\title{
El profesorado de ciencias sociales en contextos rurales de Asturias: concepciones y uso de recursos educativos
}

\author{
María Belén San Pedro Veledo, Inés López Manrique \\ Departamento de Ciencias de la Educación. Universidad de Oviedo
}

\begin{abstract}
RESUMEN
El presente trabajo tiene dos objetivos, a) examinar las concepciones de los docentes de centros rurales o en contextos rurales sobre las posibilidades del entorno cercano como espacio de aprendizaje educativo y b) comprobar el tipo de recursos que suelen utilizar en sus clases que pueden vincularse o no con las técnicas de la Historia Rural y la Place-Based Education. Se ha utilizado un cuestionario de elaboración propia que mide el uso de recursos educativos por parte del profesorado y las concepciones de los docentes con respecto a la enseñanza de las Ciencias Sociales, las posibilidades didácticas del paisaje, del medio rural y de la toponimia en el aprendizaje del alumnado, y se ha aplicado a una muestra de 29 docentes de Educación Primaria y Secundaria en centros educativos rurales del Principado de Asturias. Para conocer la fiabilidad se ha aplicado el estadístico Alfa de Cronbach. Los resultados obtenidos al realizar la correlación de Spearman, la t de Student y la d de Cohen evidencian que el profesorado tiene una concepción muy positiva del medio rural como espacio de aprendizaje, si bien manifiesta dificultades a la hora de utilizar todos los potenciales recursos educativos para la enseñanza de ese entorno.
\end{abstract}

Palabras Clave: Educación Rural, Ciencias Sociales, Historia Rural, Recursos educativos, Place-Based Education.

\section{The teachers of social sciences in rural contexts of Asturias: conceptions and use of educational resources}

\section{ABSTRACT}

This paper has two objectives: a) to examine the conceptions of teachers in rural centers or in rural settings about the possibilities of immediate environment as an area of educational learning b) check the type of resources they typically use in their classrooms that can be linked or not with the techniques of Rural History and Place-Based Education. It has been used a specifically designed questionnaire that measures the use of educational resources by teachers and conceptions of teachers regarding the teaching of Social Sciences, the educational possibilities of the landscape, rural and place names in the student learning. The questionnaire has been applied to a sample of 29 teachers of primary and secondary education in rural schools of the Principality of Asturias. For reliability, has been applied Cronbach alpha statistic. The results obtained by performing the correlation of Spearman, Student's $t$ test and Cohen's $d$ show that teachers have a very positive view of rural areas as a learning space, while they evince difficulties to use all the potential educational resources for teaching that environment.

Keywords: Rural Education, Social Sciences, Rural History, Educational Resources, Place-Based Education.

\section{Introducción}

Medio rural y Ciencias Sociales

Las Ciencias Sociales constituyen una materia troncal a nivel nacional y autonómico, tanto en la Educación Primaria, tratadas dentro de la asignatura homónima de Ciencias Sociales, como en la Educación Secundaria, por medio de la materia de Geografía e
Historia. Los contenidos referidos a estas ciencias se imparten en todos los cursos de ambas etapas, con una carga lectiva que varía entre las tres y cuatro horas dependiendo del curso en concreto. En los últimos años se ha puesto de manifiesto la necesidad de realizar un replanteamiento de la enseñanza de las Ciencias Sociales en general y de la Historia en particular (Downey \&Long, 2015) con el objeto de que el alumnado se familiarice con el "pensamiento histórico" (historical thinking) y mejore su competencia 
en la disciplina (historical literacy). Miralles, Gómez y Sánchez (2014) han analizado cómo en los exámenes de Ciencias Sociales de tercer ciclo de Educación Primaria se siguen evaluando contenidos conceptuales con un enfoque memorístico dejando a un lado las capacidades y habilidades relacionadas con la comprensión, el análisis y la relación. Es necesario en este sentido potenciar las llamadas estrategias de elaboración que consigan que los estudiantes relacionen las distintas partes del contenido a aprender y sean capaces de hacerlo también con conocimientos previos (Martín-Antón, Marugán, Catalina y Carbonero, 2013). La falta de contenidos procedimentales y actitudinales provoca que aún hoy en la enseñanza de la Historia no haya una auténtica relación entre el saber y el saber hacer. De ahí la importancia de introducir el método del historiador en el proceso de enseñanza y aprendizaje de la disciplina y de promover en el alumnado la consecución de habilidades relacionadas con la investigación en la misma.

En este contexto de cambios en los parámetros de la enseñanza debe insertarse el aprendizaje en los centros educativos rurales o Colegios Rurales Agrupados, y en los centros educativos en contextos rurales, aludiendo estos últimos a centros que se ubican en el mismo medio rural o en sus proximidades sin el carácter de enseñanza agrupada que se imparte en los primeros. Si bien en los últimos años se ha hecho patente una mejora en el uso de recursos relacionados con las Tecnologías de la Información y la Comunicación, tanto en profesorado como en el alumnado de centros rurales (Del Moral, Villalustre y Neira, 2013; Villalustre y Del Moral, 2011), la crisis económica actual y el cambio demográfico del mismo medio hace necesario el uso de otros recursos disponibles, como el propio entorno cercano.

El entorno inmediato es una fuente primaria de estudio para las Ciencias Sociales, en él se conjugan factores naturales e históricos que pueden ser analizados desde la Geografía y la Historia de modo conjunto. Mediante el análisis territorial, el registro de las formas de paisaje, la información oral y la documental se puede realizar un estudio que sirva para analizar lo general desde lo particular y viceversa (Post, 2015; Prada, Riesco y Herrero, 2013). Incluso siendo obvio que en los entornos rurales los estilos de vida y sus costumbres se están urbanizando cada vez más (Bustos, 2009), todavía es posible descubrir los restos del pasado en el paisaje actual. Durante los últimos años desde el ámbito educativo algunas voces insisten en la importancia de incluir la Historia y la cultura local en el currículo oficial, sobre todo en entornos rurales (Balarin y Benavides, 2010). García, Jiménez y Rodríguez (2009) sostienen que la Geografía y la Historia se enseñan por lo general de modo abstracto y aislado no recurriéndose a la cotidianeidad del alumnado ni a su realidad inmediata como como contenido educativo. Es necesario por tanto dotar a los estudiantes de instrumentos de investigación que fomenten el aprendizaje por indagación estudiando su entorno de manera crítica. En este sentido, la enseñanza y aprendizaje sobre el contexto local que nos rodea puede servir para pensar sobre el medio ambiente y entender las raíces históricas del lugar en el que se vive, sirviendo como un "laboratorio" (Bustos, 2009) en el que cabe una aproximación interdisciplinar, no sólo desde la Historia sino también desde la Geología, la Biología y la Antropología entre otras (Capshew, 2010). De hecho, se ha demostrado cómo los contextos rurales pueden ser ambientes idóneos para el aprendizaje de la ciencia y las disciplinas vinculadas (Avery, 2013). El alumnado en contextos rurales adquiere de forma natural a lo largo de su vida cotidiana habilidades relacionadas con la ciencia, así que utilizando como estrategia educativa el día a día de los estudiantes e involucrando al profesorado y a la comunidad se puede incrementar de manera notable el interés por las disciplinas científicas.
En Estados Unidos el programa PACERS (Program for Academic and Cultural Excellence in Rural Schools) propugna un nuevo enfoque curricular que busca vincular las aulas con sus comunidades y regiones, promoviendo incentivar la documentación del arte y la historia local por parte del profesorado (Smith, 2007). En este sentido, experiencias llevadas a cabo en Kansas ponen de manifiesto la importancia de la historia local en el medio rural, involucrando a los estudiantes en la investigación e introduciendo al mismo tiempo las TIC mediante la elaboración de podcasts relacionados. Los propios paisajes o los vestigios del pasado en el medio rural tienen la capacidad afectiva de generar recuerdos de lugares y personas en el presente. Un estudio reciente realizado en Askham (Cumbria, Reino Unido) documenta cómo la población ha interiorizado los restos del paisaje de su pasado minero, jugando un papel esencial en la propia comprensión del entorno y de su temporalidad (Wheeler, 2014).

En un momento de crisis sistémica del medio rural se hace vital lograr que los centros educativos se constituyan en puntos activos que sean capaces de conectar con el resto de la comunidad. La escuela depende del lugar en el que se ubica y tiene que ser consciente de su propia ruralidad para conseguir una conexión funcional entre comunidad y centro educativo (Hargreaves, Kvaldsund y Galton, 2009). Estudiantes, familias y profesorado tienen que desarrollar el aprecio y el conocimiento de su propia historia, de su arte, y de las circunstancias específicas de su región para conocer su pasado y pensar su futuro. Algunos estudios ponen de manifiesto la importancia de esa conexión más profunda entre escuela y medio rural, llevando a cabo proyectos que sirvan a lo local (Bargley y Hillyard, 2011; Munro, 2010). Otros estudios llevados a cabo en áreas como Educación Física, constatan los beneficios de vincular las experiencias de aula de los estudiantes con sus comunidades, resultando en niveles más profundos de aprendizaje, en el establecimiento de asociaciones comunitarias productivas y en la creación de agentes de cambio en la localidad (Alfrey y O'Connor, 2014). En las Ciencias Sociales, la implicación de la comunidad es básica a la hora del estudio y del mantenimiento de paisajes, tradiciones, lenguas, fiestas y demás elementos culturales. Un planteamiento multidisciplinar que analice las distintas realidades rurales necesita ser comprendido desde la escuela por todos los colectivos integrantes de la comunidad (Bustos, 2011).

\section{Paisaje, patrimonio rural y Place-Based Education}

Podemos afirmar que en el siglo XXI se ha producido un cambio en la concepción hacia el patrimonio y la consideración del paisaje con la introducción del concepto de Paisaje Cultural. El Convenio Europeo del Paisaje, suscrito en Florencia en el año 2000 y la Carta de la Tierra propugnada en el mismo año tienen, entre otros, el objetivo de concienciar del valor patrimonial del paisaje y consolidar en la población una conciencia de protección y respeto hacia el medio ambiente por medio de la educación. Existen investigaciones sobre proyectos educativos en ambos sentidos con muy buenos resultados: aumento de la conciencia medioambiental, apertura de las aulas hacia la comunidad, mejora del entorno y un aprendizaje más duradero y profundo (Hinojosa y Arenas, 2012; Meijles y Van Hoven, 2010). El carácter interdisciplinario del paisaje hace que por medio de su tratamiento se puedan abordar todas las competencias clave con el alumnado, por lo que es básica la formación del profesorado para conseguir una práctica correcta (Delgado, 2015). El Paisaje Cultural, definido en el Convenio Europeo del Paisaje celebrado en Florencia en el año 2000, en el artículo 1(a), como cualquier parte del territorio tal como la percibe la población, cuyo carácter sea 
el resultado de la acción y la interacción de factores naturales y/o humanos supone un auténtico cambio en la consideración hacia los paisajes. Se cambia de una concepción centrada sólo en la protección y consideración de paisajes con grandes monumentos y sitios arqueológicos y /o históricos de entidad arquitectónica, a la significación de todo tipo de paisaje al ser producto de condicionantes naturales y humanos en el tiempo largo. El Paisaje Cultural se relaciona así con la "Historia Viva" constituyendo un rico registro para la Historia Social (Taylor y Lennon, 2012). Dentro de esta consideración hacia las formas paisajísticas no tenidas tradicionalmente en cuenta juega un papel clave el paisaje rural. Se propugna ya la conservación del patrimonio agrario, integrando los valores patrimoniales en las estrategias para la sostenibilidad medioambiental, económica y social en las áreas rurales (Mitchell y Barrett, 2015), reconociendo la importancia de los paisajes culturales rurales como auténticos documentos históricos (Taylor, 1990).

En la actualidad podría decirse que los paisajes rurales están cambiando, son paisajes en transición (Kizos, Primdal, Kristensen y Busck, 2010). No obstante, aún es viable el estudio de los paisajes previos, constatando las características de cada época utilizando métodos regresivos (Cevasco, 2010). La idea no es nueva, Marc Bloch, historiador especializado en la Edad Media constató las posibilidades del estudio del mundo rural a través de una metodología científica. Durante siglos, el campo y las aldeas han sido el escenario y el motor económico de distintas colectividades. En su obra, Bloch (1978) proponía un "método regresivo" aplicado a marcos topográficos restringidos: un área determinada, una localidad, etc. El método partía del análisis regresivo de un paisaje rural desde el presente hasta el pasado. Al fin y al cabo, el Paisaje es una especie de palimpsesto formado por distintas y sucesivas capas que cada una de las sociedades ha transformado a lo largo del tiempo. Por lo tanto, para conocer cómo era el paisaje rural de una determinada época se debe ir "levantando cada capa", observando lo modificado en cada período hasta llegar a la etapa objeto de estudio. Este trabajo implica tanto la observación directa del paisaje como la utilización de fuentes escritas y toponímicas que aludan a él a lo largo de la Historia. La Historia Rural, o Agraria, ha utilizado un método propio de análisis que comparte muchos aspectos de la prospección arqueológica clásica, si bien su objetivo se centra más en conocer el desarrollo diacrónico de un determinado entorno rural mediante métodos alternativos a la intervención arqueológica. Para ello es necesaria la obtención de información mediante el estudio de fuentes primarias y secundarias, la interpretación de todo tipo de cartografía y fotografía aérea, el análisis toponímico, la observación directa mediante el trabajo de campo y la encuesta oral.

Tanto el currículo español de Educación Primaria como el de Educación Secundaria Obligatoria incluyen entre sus contenidos algunos relacionados con el estudio del entorno inmediato, los paisajes locales, así como procedimientos vinculados a la recogida de información mediante la encuesta oral, el uso de mapas y la interpretación correcta de fotografías. Por lo tanto, cobra interés en este sentido introducir esas "técnicas" vinculadas a la Historia Rural en la enseñanza obligatoria, más si cabe en contextos rurales. Las salidas de campo y el vaciado toponímico del entorno se presentan como recursos de grandes posibilidades educativas. Si bien las salidas al entorno se están utilizando en áreas como la Geografía e incluso el Arte (Marco, 2016), es necesario pensar en una auténtica educación al aire libre dentro de un marco pedagógico orientado también hacia el desarrollo sostenible del medio ambiente (Wattchow y Higgins, 2014).

Las pequeñas investigaciones del alumnado en el medio rural son un medio excelente para establecer esa conexión entre escuela y comunidad a la que aludíamos con anterioridad. El vaciado toponímico del entorno inmediato es clave en este sentido. Los nombres de lugar y la microtoponimia de una localidad pueden leerse como un texto que representa una teoría del

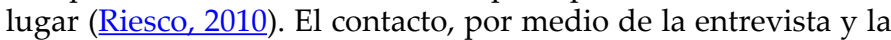
encuesta oral, con los mayores del lugar puede ser muy enriquecedor tanto para la recogida sistemática de topónimos de gran valor patrimonial como para la creación de lazos entre la escuela y la comunidad. El interés académico por la toponimia y sus posibilidades didácticas se ha reavivado en las últimas décadas, ya que los topónimos proporcionan acceso a los aspectos más complejos de la Historia, de la memoria y de la localidad proporcionando datos sobre el mundo natural, la actividad humana y la lengua (Fuchs, 2015; Heikkilä y Fondahl, 2010). Asimismo, la toponimia tiene un valor formativo multidisciplinar al reflejar procesos sociales y naturales.

Todas estas técnicas pueden relacionarse con una metodología inserta en la Place-Based Education (PBE) (Smith, 2002; Sobel, 2005). El PBE se estructura a partir del aprendizaje en torno a la historia local, la cultura, el marco geográfico, la lengua, el medio ambiente y la economía, de un modo interdisciplinar y experimental, dejando a un lado el aprendizaje memorístico y utilizando la resolución de problemas en el mundo real. No obstante, aunque las experiencias constatan una mejora significativa tanto del aprendizaje profundo como de la conciencia medioambiental (Howley, Howley, Camper, y Perko, 2011), la metodología PBE tiene sus riesgos siendo necesario realizar una lectura crítica de lo que significa "lo local" conectando lo cercano con contenidos más globales (McInerney, Smyth y Down, 2011).

Según se desprende de las diferentes experiencias educativas y de las recomendaciones bibliográficas existentes, donde se confirman los beneficios de incluir en el currículo oficial los estudios del entorno cercano, y teniendo en cuenta la escasez de trabajos donde se investigue el pensamiento del profesorado a cerca de esta temática, no es baladí contar con la opinión de los docentes para la implementación de este tipo de experiencias. El propio profesorado en contextos rurales debe reflexionar sobre sus concepciones sobre el proceso educativo para orientar sus prácticas pedagógicas hacia actividades significativas (Bachmann, Osses, y Schiefelbein, 2012).

Los principales objetivos de este estudio son los de examinar las concepciones de los docentes de centros rurales y en contextos rurales sobre las posibilidades del entorno cercano como espacio de aprendizaje educativo, y comprobar el tipo de recursos que suelen utilizar en sus clases ordinarias que pueden vincularse o no con las técnicas de la Historia Rural y el PBE. Nuestras hipótesis de partida son:

1. El profesorado de centros rurales y en contextos rurales tiene concepciones positivas hacia el uso didáctico del entorno cercano, sobre todo del propio medio rural.

2. El profesorado de centros rurales y en contextos rurales no está explotando todos los recursos relacionados con el uso didáctico del medio rural.

\section{Metodología}

\section{Participantes}

Los participantes en este estudio han sido 29 docentes de las asignaturas de Ciencias Sociales y de Geografía e Historia (11 de Educación Primaria y 18 de Educación Secundaria), que imparten docencia en Centros Públicos del entorno rural del Principado de Asturias. En cuanto al género, 12 son varones y 18 muje- 
res. Se ha dividido a los participantes en dos grupos de edades, 18 tienen menos de 45 años (62\%) y 11 son mayores de 45 años $(48 \%)$. Otra cuestión que se ha tenido en cuenta ha sido los años de experiencia docente y los resultados muestran que el $41,4 \%$ lleva menos de 10 años, el $24,1 \%$ de 10 a 20 , el $13,8 \%$ de 20 a 30 y el $20,7 \%$, más de 30 años (41.4\% menos de 10 años y el $58.6 \%$ más de 10).

\section{Instrumento}

El instrumento de medida utilizado ha sido un cuestionario de elaboración personal sobre recursos didácticos en el medio rural aplicables en las asignaturas de Ciencias Sociales y Geografía e Historia (RDMR-GH) formado por tres grupos de preguntas, grupo A preguntas personales y de situación laboral con ocho ítems; grupo B, llamado "Concepciones" y formado por veinte variables relacionadas con las concepciones del profesorado acerca de la enseñanza de la Geografía y de la Historia, posibilidades didácticas del estudio del Paisaje, posibilidades didácticas del propio medio rural y posibilidades didácticas del uso de la toponimia. Los ítems se responden en una escala tipo Likert de 1 a 5 , siendo 1 totalmente en desacuerdo y 5 totalmente de acuerdo con las cuestiones presentadas (Ejemplo de ítems de este grupo: afirmación “B01. La enseñanza de la Geografía y la Historia debe tener una vertiente más práctica para facilitar el aprendizaje del alumnado", y la afirmación "B15. Es positivo que el alumnado realice trabajos de investigación en el medio rural mediante la realización de entrevistas a sus habitantes").

Se han agrupado los ítems del grupo B sobre "Concepciones" en cuatro dimensiones a las que se han denominado "Enseñanza", "Paisaje", "Medio Rural" y "Toponimia". La dimensión "Enseñanza" mide cuáles son las concepciones del profesorado en cuanto a los estilos de enseñanza de las Ciencias Sociales y el peso de la práctica frente a la teoría en la disciplina. La segunda dimensión, "Paisaje", mide las concepciones de los docentes con respecto a la importancia y las posibilidades del paisaje como instrumento educativo. Bajo la etiqueta "Medio Rural" agrupamos un conjunto de variables que miden las concepciones del profesorado en cuanto a las posibilidades didácticas del medio rural y la viabilidad de emplearlo en las clases ordinarias. Por último, en la dimensión "Toponimia" se recogen aquellas variables que miden la adecuación de su uso como recurso didáctico y la facilidad y/o conveniencia de integrarla en las aulas.

El grupo C está formado por 19 variables relacionadas con la utilización de recursos educativos por parte del profesorado, usando también una escala Likert de 1 a 5 siendo 1 nada y 5 mucho. Todas las variables referidas a recursos se recogen en la Tabla 1.

El cuestionario fue revisado por quince expertos, cinco docentes universitarios, cinco docentes de Educación Primaria y cinco docentes de Educación Secundaria que tras realizar las modificaciones sugeridas dieron su aprobación.

\section{Procedimiento}

El cuestionario fue enviado por medio de correo electrónico a 120 centros de Educación Primaria y Educación Secundaria del Principado de Asturias, todos ellos centros rurales o en contextos rurales fuera del área de influencia de las tres principales ciudades de la región. Se solicitó la colaboración de los docentes que impartiesen asignaturas relacionadas con las Ciencias Sociales (Ciencias Sociales, Geografía, Historia e Historia del Arte), explicando los objetivos del estudio y asegurando el completo anonimato en las respuestas. Finalmente, fueron 29 los docentes que cubrieron el cuestionario de forma satisfactoria al no haber dado la posibilidad de envío de respuestas con preguntas en blanco.

\section{Análisis estadístico}

Se ha realizado un examen exploratorio de los datos de las variables del grupo C "Recursos", segmentando los resultados por docentes de Educación Primaria y Educación Secundaria. Para ello se han aplicado pruebas de tendencia central y dispersión.

A continuación, se han agrupado los ítems del grupo B, "Concepciones", en cuatro dimensiones. Para determinar el nivel de fiabilidad se ha aplicado el estadístico Alfa de Cronbach.

Con el fin de determinar si existía correlación bivariable estadísticamente significativa entre las cuatro dimensiones, se ha aplicado la prueba Rho de Spearman. Para comprobar si en las cuatro dimensiones estudiadas la diferencia de medias en función del sexo y del nivel dónde imparten docencia es estadísticamente significativa, y si el tamaño del efecto es aceptable, se han aplicado los estadísticos $t$ de Student y $d$ de Cohen.

El tratamiento y análisis de los datos obtenidos se ha formalizado utilizando el paquete estadístico SPSS.22 para Windows y el G*Power 3.1.

\section{Resultados}

El 96,5 de los docentes consideran que el paisaje rural es parte de nuestro patrimonio cultural. Asimismo, el 79,3\% de los docentes consultados considera que la enseñanza de las Ciencias Sociales debería tener una vertiente más práctica, que el estudio del paisaje debería ser obligatorio en el currículo oficial y que es importante y conveniente que el alumnado de centros rurales o en contextos rurales realice prácticas en el medio rural. De hecho, el 93,1 \% de los encuestados considera que es positivo que los estudiantes lleven a cabo pequeños trabajos de investigación en el medio rural mediante la realización de entrevistas a sus habitantes y la observación directa. No obstante, tan sólo el 20,6 \% de los docentes manifiesta que le resulta sencillo diseñar nuevas prácticas cada curso para la disciplina. En este sentido también es significativo que el $78 \%$ de los docentes considere el estudio de parcelarios rurales como potencial recurso educativo y que el 89,6\% crea que estas salidas de campo contribuyen a la formación cívica del alumnado. Por otro lado, llama la atención que el $89,7 \%$ del profesorado contemple la toponimia como un recurso educativo de primer nivel y sin embargo sólo un 44,8 \% considere que es fácil integrarla en las clases ordinarias.

Para conocer la fiabilidad se ha aplicado el estadístico Alfa de Cronbach. Los resultados muestran un buen ajuste en tres de las dimensiones y en la totalidad de los ítems. (Ver tabla 2).

Los resultados de la prueba estadística Rho de Spearman muestran que existe una correlación estadísticamente significativa en un 99\% $(\mathrm{p}<, 01)$ entre "Medio Rural" con "Paisaje y "Toponimia" (0,482 y 0,668 respectivamente), y entre "Toponimia" y "Paisaje" (0,508), y con un 95\% ( $\mathrm{p}<.05)$ entre "Enseñanza" y "Medio Rural" (0,407). (Ver tabla 2).

Seguidamente se procede a comparar las diferencias o dependencia de las variables género, edad, nivel educativo donde imparten docencia y años de dedicación docente con las cuatro dimensiones de "Concepciones"; se ha aplicado el estadístico $t$ de Student, para conocer si las diferencias de medias son estadísticamente significativas, y la $d$ de Cohen para conocer el tamaño del efecto.

Los resultados en cuanto a la diferencia de medias en referencia al género muestran que aparecen diferencias estadísticamen- 
Tabla 1. Medidas de tendencia central y dispersión de las variables "Recursos".

\begin{tabular}{|c|c|c|c|c|c|}
\hline \multicolumn{2}{|l|}{ Variables (RECURSOS) } & \multirow{2}{*}{$\begin{array}{c}\mathrm{M} \\
3,52 \\
3,09 \\
3,78\end{array}$} & \multirow{2}{*}{$\begin{array}{c}\text { SD } \\
1,022 \\
1,044 \\
0,943\end{array}$} & \multirow{2}{*}{$\begin{array}{c}\text { Asim. } \\
-0,158 \\
-0,213 \\
0,021\end{array}$} & \multirow{2}{*}{$\begin{array}{c}\text { Curt. } \\
-0,061 \\
1,253 \\
-1,096\end{array}$} \\
\hline Fuentes arqueológicas (objetos o fotos de objetos, visitas a yacimientos, etc.) & $\begin{array}{l}\text { Totalidad } \\
\text { Prof. Primaria } \\
\text { Prof. Secundaria }\end{array}$ & & & & \\
\hline Fuentes toponímicas (nombres de lugares) & $\begin{array}{l}\text { Totalidad } \\
\text { Prof. Primaria } \\
\text { Prof. Secundaria }\end{array}$ & $\begin{array}{l}3,41 \\
3,09 \\
3,61\end{array}$ & $\begin{array}{l}1,086 \\
1,044 \\
1,092\end{array}$ & $\begin{array}{l}-, 0032 \\
0,431 \\
-0,319\end{array}$ & $\begin{array}{l}-1^{\prime} 284 \\
-0,932 \\
-1,114\end{array}$ \\
\hline Fuentes orales & $\begin{array}{l}\text { Totalidad } \\
\text { Prof. Primaria } \\
\text { Prof. Secundaria }\end{array}$ & $\begin{array}{l}3,07 \\
3,36 \\
2,89\end{array}$ & $\begin{array}{l}1,193 \\
0,924 \\
1,323\end{array}$ & $\begin{array}{l}-0,141 \\
0,951 \\
-0,116\end{array}$ & $\begin{array}{c}-0,368 \\
0,373 \\
-0,935\end{array}$ \\
\hline Fuentes documentales (documentos, textos y relatos históricos) & $\begin{array}{l}\text { Totalidad } \\
\text { Prof. Primaria } \\
\text { Prof. Secundaria }\end{array}$ & $\begin{array}{l}4,07 \\
3,73 \\
4,28\end{array}$ & $\begin{array}{l}0,884 \\
0,905 \\
0,826\end{array}$ & $\begin{array}{l}-0,808 \\
-0,344 \\
-1,297\end{array}$ & $\begin{array}{c}0,235 \\
-0,054 \\
2,103\end{array}$ \\
\hline Fuentes artísticas (cuadros, edificios, esculturas, etc.) & $\begin{array}{l}\text { Totalidad } \\
\text { Prof. Primaria } \\
\text { Prof. Secundaria }\end{array}$ & $\begin{array}{l}4,17 \\
3,64 \\
4,50\end{array}$ & $\begin{array}{l}0,759 \\
0,674 \\
0,618\end{array}$ & $\begin{array}{l}-0,306 \\
0,593 \\
-0,840\end{array}$ & $\begin{array}{l}-1,151 \\
-0,293 \\
-0,101\end{array}$ \\
\hline Libros de texto & $\begin{array}{l}\text { Totalidad } \\
\text { Prof. Primaria } \\
\text { Prof. Secundaria }\end{array}$ & $\begin{array}{l}4,10 \\
4,18 \\
4,06\end{array}$ & $\begin{array}{l}1,081 \\
0,751 \\
1,259\end{array}$ & $\begin{array}{l}-1,131 \\
-0,329 \\
-1,111\end{array}$ & $\begin{array}{c}0,880 \\
-0,878 \\
0,323\end{array}$ \\
\hline Datos estadísticos (ej: datos de la web del SADEI) & $\begin{array}{l}\text { Totalidad } \\
\text { Prof. Primaria } \\
\text { Prof. Secundaria }\end{array}$ & $\begin{array}{l}3,21 \\
2,73 \\
3,50\end{array}$ & $\begin{array}{l}1,114 \\
1,421 \\
0,786\end{array}$ & $\begin{array}{c}-0,273 \\
0,586 \\
-0,409\end{array}$ & $\begin{array}{l}-0,617 \\
-0,795 \\
-0,069\end{array}$ \\
\hline Mapas & $\begin{array}{l}\text { Totalidad } \\
\text { Prof. Primaria } \\
\text { Prof. Secundaria }\end{array}$ & $\begin{array}{l}4,66 \\
4,45 \\
4,78\end{array}$ & $\begin{array}{l}0,553 \\
0,688 \\
0,428\end{array}$ & $\begin{array}{l}-1,355 \\
-0,932 \\
-1,461\end{array}$ & $\begin{array}{l}1,044 \\
0,081 \\
0,137\end{array}$ \\
\hline Ortofotomapas & $\begin{array}{l}\text { Totalidad } \\
\text { Prof. Primaria } \\
\text { Prof. Secundaria }\end{array}$ & $\begin{array}{l}2,69 \\
2,36 \\
2,89\end{array}$ & $\begin{array}{l}1,198 \\
1,027 \\
1,278\end{array}$ & $\begin{array}{l}-0,149 \\
-0,229 \\
-0,340\end{array}$ & $\begin{array}{l}-1,050 \\
-1,134 \\
-1,064\end{array}$ \\
\hline Cartografía digital & $\begin{array}{l}\text { Totalidad } \\
\text { Prof. Primaria } \\
\text { Prof. Secundaria }\end{array}$ & $\begin{array}{l}2,97 \\
2,82 \\
3,06\end{array}$ & $\begin{array}{l}1,149 \\
0,982 \\
1,259\end{array}$ & $\begin{array}{l}-0,232 \\
-0,346 \\
-0,315\end{array}$ & $\begin{array}{l}-0,621 \\
-0,587 \\
-0,656\end{array}$ \\
\hline Webs cartográficas (Google Earth, Google Maps, SIgPac, etc.) & $\begin{array}{l}\text { Totalidad } \\
\text { Prof. Primaria } \\
\text { Prof. Secundaria }\end{array}$ & $\begin{array}{l}3,41 \\
3,55 \\
3,33\end{array}$ & $\begin{array}{l}1,053 \\
1,128 \\
1,029\end{array}$ & $\begin{array}{c}0,047 \\
0,118 \\
-0,040\end{array}$ & $\begin{array}{l}-1,156 \\
-1,306 \\
-1,170\end{array}$ \\
\hline S. I. G. (Sistemas de Información Geográficos) & $\begin{array}{l}\text { Totalidad } \\
\text { Prof. Primaria } \\
\text { Prof. Secundaria }\end{array}$ & $\begin{array}{l}2,48 \\
2,55 \\
2,44\end{array}$ & $\begin{array}{l}1,214 \\
1,036 \\
1,338\end{array}$ & $\begin{array}{c}0,365 \\
-0,147 \\
0,547\end{array}$ & $\begin{array}{l}-0,578 \\
-0,853 \\
-0,529\end{array}$ \\
\hline Fotografías aéreas & $\begin{array}{l}\text { Totalidad } \\
\text { Prof. Primaria } \\
\text { Prof. Secundaria }\end{array}$ & $\begin{array}{l}2,86 \\
2,73 \\
2,94\end{array}$ & $\begin{array}{l}1,026 \\
1,191 \\
0,938\end{array}$ & $\begin{array}{l}-0,133 \\
0,205 \\
-0,362\end{array}$ & $\begin{array}{r}-0,447 \\
0,181 \\
-0,823\end{array}$ \\
\hline Fotografías actuales o fotografías históricas & $\begin{array}{l}\text { Totalidad } \\
\text { Prof. Primaria } \\
\text { Prof. Secundaria }\end{array}$ & $\begin{array}{l}3,90 \\
3,64 \\
4,06\end{array}$ & $\begin{array}{l}1,012 \\
0,924 \\
1,056\end{array}$ & $\begin{array}{l}-0,890 \\
-0,023 \\
-1,472\end{array}$ & $\begin{array}{c}0,916 \\
-0,448 \\
2,899\end{array}$ \\
\hline Documentales didácticos & $\begin{array}{l}\text { Totalidad } \\
\text { Prof. Primaria } \\
\text { Prof. Secundaria }\end{array}$ & $\begin{array}{l}3,76 \\
3,45 \\
3,94\end{array}$ & $\begin{array}{l}1,123 \\
0,934 \\
1,211\end{array}$ & $\begin{array}{l}-0,786 \\
-0,290 \\
-1,223\end{array}$ & $\begin{array}{c}-0,107 \\
-0,501 \\
0,796\end{array}$ \\
\hline Películas y/o series & $\begin{array}{l}\text { Totalidad } \\
\text { Prof. Primaria } \\
\text { Prof. Secundaria }\end{array}$ & $\begin{array}{l}3,45 \\
3,36 \\
3,50\end{array}$ & $\begin{array}{l}1,021 \\
0,924 \\
1,098\end{array}$ & $\begin{array}{l}-0,391 \\
0,023 \\
-0,600\end{array}$ & $\begin{array}{c}-0,166 \\
-0,448 \\
0,142\end{array}$ \\
\hline Videojuegos & $\begin{array}{l}\text { Totalidad } \\
\text { Prof. Primaria } \\
\text { Prof. Secundaria }\end{array}$ & $\begin{array}{l}1,79 \\
1,91 \\
1,72\end{array}$ & $\begin{array}{l}1,013 \\
1,136 \\
0,958\end{array}$ & $\begin{array}{l}0,888 \\
0,711 \\
1,083\end{array}$ & $\begin{array}{c}-0,560 \\
-1,146 \\
0,131\end{array}$ \\
\hline Salidas de campo & $\begin{array}{l}\text { Totalidad } \\
\text { Prof. Primaria } \\
\text { Prof. Secundaria }\end{array}$ & $\begin{array}{l}3,28 \\
3,73 \\
3,00\end{array}$ & $\begin{array}{l}1,066 \\
0,905 \\
1,085\end{array}$ & $\begin{array}{c}-0,219 \\
-0,344 \\
0,000\end{array}$ & $\begin{array}{l}-0,777 \\
-0,054 \\
-0,948\end{array}$ \\
\hline
\end{tabular}


Tabla 2. Correlaciones bivariadas y alfa de Cronbach

\begin{tabular}{llcccc}
\hline & & Enseñanza & M. Rural & Toponimia & Paisaje \\
\hline & Enseñanza & 1,000 & & & \\
& M. Rural & $0,407^{*}$ & 1,000 & & \\
\multirow{2}{*}{ Rho de Spearman } & Toponimia & 0,110 & $0,665^{* *}$ & 1,000 & $0,508^{* *}$ \\
& Paisaje (3 ítem) & 0,256 & $0,482^{* *}$ & 0,000 \\
\hline Alfa de Cronbach & 0,657 & 0,846 & 0,852 & 0,841 & 0,624 \\
\hline
\end{tabular}

* La correlación es significativa al nivel 0,05 (bilateral).

**. La correlación es significativa al nivel 0,01 (bilateral).

te significativas en la dimensión correspondiente al Medio Rural $(t=-3,376, p=0,02)$ presentando un tamaño del efecto muy bueno $(d=1,33)$, siendo las mujeres las que otorgan un valor más alto $(\mathrm{DM}=0,673)$. En el resto de dimensiones no aparecen diferencias estadísticamente significativas.

En relación al nivel donde imparten docencia aparecen diferencias de medias estadísticamente significativas en la dimensión Paisaje $(t=-3,376, p=0,033)$ con un tamaño del efecto bueno $(d=0,85)$ puntuando más alto el profesorado de Secundaria (DM $=-0,449)$; también muestran diferencias estadísticamente significativas en la dimesnión Toponimia $(t=-2,177, p=0,04)$ y un tamaño del efecto bueno $(d=0,86)$, siendo la puntuación más alta también la del profesorado de Secundaria $(\mathrm{DM}=0,658)$. ( $\underline{\text { Ver }}$ tabla 3$)$.

Con respecto a las diferencias de medias en relación a la edad de los participantes y los años de dedicación docente, no ha aparecido ninguna significación estadística.

La mayor parte de los ítems de las variables agrupadas bajo la denominación "Recursos" (grupo C) se relacionan con las distintas técnicas vinculadas a la metodología de la Historia Rural y el PBE, excepto el uso de libro de texto, documentales, películas y/o series y videojuegos.

La puntuación media o central es de 3 puntos, por lo que siempre que la media sea superior a tres indica un uso intensivo del recurso, mientras que si está por debajo de 3 el uso es escaso. Uno de los recursos más utilizado por parte del profesorado de centros rurales o de centros en contextos rurales sigue siendo el libro de texto; el $72,4 \%$ de los docentes afirma usarlo bastante o mucho, tanto en Educación Primaria $(\mathrm{M}=4,18)$ como en Educación Secundaria $(M=4,06)$, mientras que sólo el $48,2 \%$ afirma utilizar mucho o bastante las salidas de campo, en Educación Primaria $(M=3,73)$ y Educación Secundaria $(M=3,00)$. No obstante, en uno de los ítems de la dimensión "medio rural" del grupo B, el 65,5\% de los docentes veía viable realizar salidas al entorno rural próximo con motivos didácticos. Tan sólo tres de ellos explicaban la no viabilidad por problemas en cuanto a la gestión de menores, problemas de horario o la imposibilidad de organizar salidas de campo que no estuviesen definidas al principio de curso.

Un aspecto positivo a destacar es la constatación del uso intensivo de mapas como recurso educativo por parte del profesorado $(M=4,66)$, sobre todo en el caso de los docentes de Educación Secundaria. El resto de recursos vinculados con la Historia Rural y el PBE tienen un uso medio en las aulas rurales, siendo los más bajos el uso de ortofotomapas, cartografía digital, Sistemas de Información Geográficos (SIG) y fotografías aéreas. Pese a su bajo uso, sí podemos establecer que el profesorado de Educación Secundaria supera al de Educación Primaria en el uso de la cartografía digital, los ortofotomapas y las fotografías aéreas mientras que el de Educación Primaria tiene una media más alta $(\mathrm{M}=2,55)$ frente al de Secundaria en la utilización de un recurso tan complejo como los SIG.

Como decíamos, el resto de recursos vinculados a la Historia Rural y al PBE tienen un uso medio, con una media mayor en el caso de los docentes de Educación Secundaria excepto en las variables webs cartográficas $(\mathrm{M}=3,55$, de Educación Primaria frente a $M=3,33$ de Secundaria), y salidas de campo $(M=3,73$, de Educación Primaria frente a $M=3,00$ de Secundaria). En cuanto a recursos no vinculados con la Historia Rural y el PBE (documentales, películas y/o series, y videojuegos), también tienen un uso

Tabla 3. Análisis univariado para comparación de medias de las dimensiones de "Concepciones" en relación al género y a la etapa donde imparten docencia.

\begin{tabular}{|c|c|c|c|c|c|c|}
\hline & & F & $\begin{array}{l}\text { e St } \\
\text { gl }\end{array}$ & $\mathrm{p}$ & $\begin{array}{c}\text { D de Cohen } \\
\mathrm{d}\end{array}$ & $\begin{array}{l}\text { Diferencia de medias } \\
\text { DM }\end{array}$ \\
\hline \multicolumn{7}{|l|}{ Género: } \\
\hline \multicolumn{7}{|l|}{ Varones } \\
\hline \multirow[t]{2}{*}{-Mujeres } & M. Rural & $-3,376$ & 27 & 0,002 & 1,33 & $-0,673$ \\
\hline & Paisaje & $-2,251$ & 27 & 0,033 & 0,85 & $-0,449$ \\
\hline \multicolumn{7}{|l|}{ Etapa: } \\
\hline \multicolumn{7}{|l|}{ Primaria } \\
\hline -Secundaria & Toponimia & 2,231 & 27 & 0,034 & 0,86 & $-0,658$ \\
\hline
\end{tabular}


medio, siendo el más alto el de documentales $(\mathrm{M}=3,76)$ y el más bajo el de videojuegos $(M=1,79)$, este último más alto entre los docentes de Educación Primaria. (Ver resultados de tendencia central y dispersión en la tabla 1).

\section{Discusión y conclusiones}

Como se indicaba previamente, los objetivos de este trabajo consistían en explorar las concepciones de los docentes de centros rurales y en contextos rurales sobre las posibilidades del entorno cercano como espacio de aprendizaje educativo, y comprobar el tipo de recursos que utilizaban en sus clases ordinarias y si estos podían vincularse o no con las técnicas de la Historia Rural y el PBE. Las hipótesis de partida parecen corroborarse al constatarse que el profesorado tiene una concepción muy positiva del entorno rural como espacio potencial de aprendizaje, si bien manifiesta dificultades a la hora de utilizar todos los potenciales recursos educativos para la enseñanza de ese medio.

No hay aún suficientes estudios que traten las concepciones del profesorado de Ciencias Sociales en Educación Primaria y Secundaria acerca del uso del PBE, y concretamente de técnicas asociadas a la Historia Rural. La investigación confirma que el profesor sigue siendo el mayor recurso educativo junto con el libro de texto, lo que está en consonancia con otros trabajos que abordan este tema (Boix y Bustos, 2014; García, 2015). Algunos estudios evidencian que los docentes de centros educativos rurales han sido más innovadores tradicionalmente que los docentes de centros urbanos (Hargreaves, 2009:120). Bachmann y Meneses (2012) afirman que el profesorado rural intenta innovar teniendo en cuenta la necesidad de considerar el contexto sociocultural, y el patrimonio histórico y natural del lugar. Algunas investigaciones llevadas a cabo en Andalucía con los docentes de escuelas rurales (Boix y Bustos, 2014; Bustos, 2014) revelan cómo los docentes se inclinan por actividades contextualizadas con recursos propios del medio rural vinculadas a estrategias didácticas activas (investigación en el medio). Los resultados obtenidos ratifican estos datos en cuanto a las concepciones de los docentes sobre la potencialidad del entorno rural como espacio educativo, no en cuanto al uso habitual de recursos para el estudio didáctico del medio.

Por otro lado, Cuenca (2003) manifestaba en un estudio que las concepciones del profesorado a la hora de determinar referentes patrimoniales se basaba en criterios de tipo histórico. Este trabajo refleja que la consideración del profesorado en cuanto a qué es patrimonio ha avanzado significativamente desde esa fecha. El profesorado participante en el estudio cataloga el paisaje rural como patrimonio cultural. En cuanto a la toponimia, el trabajo corrobora el estudio de Villalón (2013) que afirma que los docentes tienen una insuficiente base formativa de la cultura toponímica local en el orden conceptual, procedimental y actitudinal. Si bien el profesorado consultado en este trabajo es consciente del potencial educativo de la toponimia, la mitad de los encuestados admite dificultades a la hora de integrarla en sus clases.

La principal limitación del presente estudio se encuentra en la escasa participación del profesorado consultado, perteneciente a centros educativos rurales y en contextos rurales. Aunque los resultados obtenidos revelan ciertas cuestiones de interés, sería necesario contar con una muestra mayor para lograr que estos sean generalizables. El siguiente paso consistiría en aplicar el cuestionario a una población mayor de docentes de diversas comunidades autónomas con el fin de, por una parte, validar el instrumento y contrastar, por otra, los resultados derivados. Igualmente, el estudio genera a su vez otros interrogantes que necesitan ser atendidos. Por un lado, es necesario comprobar si el bajo uso de recursos vinculados a las TIC (ortofotomapas, cartografía digital, SIG y fotografías aéreas) responde a carencias formativas del profesorado rural o bien la dificultad se encuentra en la disponibilidad y acceso a los mismos. Como línea de continuación y mejora, sería necesario implementar experiencias educativas basadas en la Historia Rural y el PBE en algunos centros ubicados en entorno rurales para corroborar su funcionamiento.

\section{Referencias bibliográficas}

Alfrey, L. y O'Connor, J. (2014). Through community: Connecting classrooms to community. En Wattchow, B., Alfrey, L., Cutter-Mackenzie, A., O'Connor, J., Brown, T., y Jeanes, R. (Eds.), The socioecological educator: A 21st century renewal of physical, health, environment and outdoor education, (pp. 109124). Dordrecht: Springer.

Avery, L. M. (2013). Rural Science Education: Valuing Local Knowledge. Theory into Practice, 52(1), 28-35.

Bachmann D. V., y Meneses Clavero, P. (2012). Construcción de una escala para medir creencias acerca del proceso educativo en profesores rurales, CAPE-R. Liberabit, 18(2), 183-193.

Bachmann, D. V., Osses, S., y Schiefelbein, E. (2012). Las Creencias de los profesores rurales: una tarea pendiente para la investigación educativa. Estudios Pedagógicos, 38(1), 297-310.

Bagley, C., y Hillyard, S. (2011). Village schools in England: At the heart of their community? Australian Journal of Education, 55 (1), 37-49.

Balarin, M. y Benavides, M. (2010). Curriculum reform and the displacement of knowledge in Peruvian rural secondary schools: exploring the unintended local consequences of global education policies. Compare, 40(3), 311-325.

Bloch, M. (1978). La historia rural francesa: caracteres originales. Barcelona: Crítica.

Brown, T., y Jeanes, R. (Eds.) (2014). The socioecological educator: A 21st century renewal of physical, health, environment and outdoor education. Dordrecht: Springer.

Bustos Jiménez, A. (2007). Enseñar en la escuela rural aprendiendo a hacerlo. Profesorado. Revista de currículum y formación del profesorado. Disponible en http://www.ugr.es/local/recfpro/ rev113COL5.pdf - Fecha de consulta 21-05-2016.

Bustos Jiménez, A. (2009). Valoraciones del profesorado de escuela rural sobre el entorno presente. Revista Iberoamericana de Educación, 48(6), 1-11.

Bustos Jiménez, A. (2011). Escuelas rurales y educación democrática. La oportunidad de la participación comunitaria. REIFOP, 14 (2), 105-114. Disponible en: http://www.aufop.com/ aufop/uploaded files/articulos/1311954573.pdf - Fecha de consulta 21-05-2016.

Bustos Jiménez, A. (2014). La didáctica multigrado y las aulas rurales: perspectivas y datos para su análisis. Innovación Educativa, 24, 119-131.

Capshew, J. H. (2010). Learning in place: The campus as ecosystem. En Reynolds, H. L., Brondizio, E. S., Robinson, J. M. (Eds.), Teaching environmental literacy: Across campus and across the curriculum, (pp. 130-134). Bloomington: Indiana University Press.

Cevasco, R. (2010). Environmental heritage of a past cultural landscape: Alder woods in the upper aveto valley of the Northwestern apennines. En Armiero, M., y Hall, M. (Eds.), Nature and History in Modern Italy, (pp. 126-140). Athens: Ohio University Press.

Consejo de Europa (2000). Convenio Europeo del Paisaje. Florencia. Disponible en http://www.mecd.gob.es/cultura-mecd/dms/ 
mecd/cultura-mecd/areas-cultura/patrimonio/Convenio europeo paisaje.pdf - Fecha de consulta 21-05-2016.

Cuenca López, J. M. (2003). Análisis de concepciones sobre la enseñanza del Patrimonio en la Educación Obligatoria. Enseñanza de las Ciencias Sociales, 2, 37-45.

Del Moral Pérez, M. A., Villalustre Martínez, L., y Neira Piñeiro, M. del R. (2013). Oportunidades de las TIC para la innovación educativa en las escuelas rurales de Asturias. Aula Abierta, 42(1), 61-67.

Delgado Huertos, E. (2015). El paisaje en la formación de maestros, un recurso educativo de alto interés para la Educación Primaria. Tabanque. Revista Pedagógica, 28, 117-138.

Downey, M. T., y Long, K. A. (2016). Teaching for a Historical Literacy: Building knowledge in the History clasroom. Nueva York: Routledge.

Fuchs, S. (2015). History and heritage of two Midwestern towns: A toponymic material approach. Journal of Historical Geography, 48(1), 11-25.

García, A., Jiménez, J., y Rodríguez, E. (2009). La enseñanza de la Geografía e Historia desde la localidad. Geoenseñanza, 14(1), 109-150.

García Prieto, F. J. (2015). Escuela, medio rural y diversidad cultural en un contexto global: currículum, materiales didácticos y práctica docente de Conocimiento del Medio: situación, límites y posibilidades en centros onubenses. Tesis doctoral disponible en http:// rabida.uhu.es/dspace/handle/10272/11440 - Fecha de consulta 21-05-2016.

Hargreaves, L. M. (2009). Respect and responsibility: Review of research on small rural schools in England. International Journal of Educational Research, 48(2), 117-128.

Hargreaves, L., Kvaldsund, R., y Galton, M. (2009). Reviews of research on rural schools and their communities in British and Nordic countries: Analytical perspectives and cultural meaning. International Journal of Education Research, 48 (2), 80 88.

Heikkilä, K., y Fondahl, G. (2010). Indigenous Toponyms as Pedagogical Tools: Reflections from Research with Tl'azt'en Nation, British Columbia. Fennia, 188(1), 105-122.

Hinojosa, E. F., y Arenas, M. (2012). Impacto de experiencias educativas basadas en la Carta de la Tierra. Aula Abierta, 40(1), 145-158.

Howley, A., Howley, M., Camper, C., y Perko, H. (2011). Place-Based Education at Island Community School. The Journal of Environmental Education, 42(4), 216-236.

Kizos, T., Primdahl, J., Kristensen, L. S., y Busck, A. G. (2010). Introduction: Landscape change and rural development. Landscape Research, 35(6), 571-576.

McInerney, P., Smyth, J., y Down, B. (2011). 'Coming to a place near you?' The politics and possibilities of a critical pedagogy of place-based education. Asia-Pacific Journal of Teacher Education, 39(1), 3-16.

Marco Mallent, M. (2016). Salir del aula: prácticas docentes alternativas para el grado en Bellas Artes. Dedica. Revista de Educaçao e Humanidades, 10 (2016), 113-125.

Martín-Antón, L. J., Marugán, M., Catalina, J. J., y Carbonero, M. A. (2013). Estrategias de aprendizaje de elaboración. Entrenamientos y Programas. Aula Abierta, 41(1), 49-62.

Meijles, E. y Van Hoven, B. (2010). Using the Rural Atelier as an Educational Method in Landscape Studies. Journal of Geography in Higher Education, 34(4), 541-560.

Miralles Martínez, P., Gómez Carrasco, C. J. y Sánchez Ibáñez, R. (2014). Dime qué preguntas y te diré qué evalúas y enseñas. Análisis de los exámenes de Ciencias Sociales en tercer ciclo de Educación Primaria. Aula Abierta, 42(2), 83-89.

Mitchell, N. J., y Barret, B. (2015). Heritage Values and Agricultural Landscapes: Towards a New Synthesis. Landscape Research, 40(6), 701-716.

Munro, L. (2010). What about history? Lessons from seven years with project-based learning. Teaching History, 138, 48-52.

Post, C. W. (2015). Seeing the past in the present through archives and the landscape. En Hanna, S. P., Potter, A. E., Modlin, E. A., Carter, P., y Butler, D. L. (Eds.), Social memory and heritage tourism methodologies, (pp. 189-209). Nueva York: Routledge.

Prada Llorente, E. I., Riesco Chueca, P., y Herrero Tejedor, T. (2013). Paisaje e imagen: formas y herencias en la construcción cultural del territorio agrario. Estudios Geográficos, 74(275), 557-583.

Riesco Chueca, P. (2010). Nombres en el paisaje: La toponimia, fuente de conocimiento y aprecio del territorio. Cuadernos Geográficos, 46(1), 7-34.

Smith, G. (2007). Grounding learning in place. World Watch, 20(2), 20-24.

Smith, G. (2002). Place-based education: Learning to be where we are. Phi Delta Kappan, 83, 584-594.

Sobel, D. (2005). Place-based education: Connecting classrooms $\mathcal{E}$ communities. Great Barrington, MA: Orion Society.

Taylor, K., y Lennon, J. L. (2012). Managing cultural landscapes. New York: Routledge.

Taylor, K. (1990). Historic cultural landscapes and emerging heritage values: And Australian perspective. Landscape Research, 15(2), 12-18.

Villalón, G. (2013). Estrategia para formación de la cultura toponímica local desde el proceso educativo escolar en secundaria básica. Revista Iberoamericana de Evaluación Educativa, 6(1), 77-94.

Villalustre, L., y Del Moral, M. A. (2011). Competencias genéricas desarrolladas por los estudiantes con las e-actividades de $R u$ ralnet. Aula Abierta, 39(2), 35-46. 